\title{
EGS \\ Theoretical Considerations for Improving the Pulse Power of a Battery through the Addition of a Second Electrochemically Active Material
}

\author{
K. W. Knehr ${ }^{*, z}$ and Alan C. West** \\ Department of Chemical Engineering, Columbia University, New York, New York 10027, USA
}

\begin{abstract}
Porous electrode theory is used to conduct case studies for when the addition of a second electrochemically active material can improve the pulse-power performance of an electrode. Case studies are conducted for the positive electrode of a sodium metal-halide battery and the graphite negative electrode of a lithium "rocking chair" battery. The replacement of a fraction of the nickel chloride capacity with iron chloride in a sodium metal-halide electrode and the replacement of a fraction of the graphite capacity with carbon black in a lithium-ion negative electrode were both predicted to increase the maximum pulse power by up to $40 \%$. In general, whether or not a second electrochemically active material increases the pulse power depends on the relative importance of ohmic-to-charge transfer resistances within the porous structure, the capacity fraction of the second electrochemically active material, and the kinetic and thermodynamic parameters of the two active materials.

(C) The Author(s) 2016. Published by ECS. This is an open access article distributed under the terms of the Creative Commons Attribution 4.0 License (CC BY, http://creativecommons.org/licenses/by/4.0/), which permits unrestricted reuse of the work in any medium, provided the original work is properly cited. [DOI: 10.1149/2.0621608jes] All rights reserved.
\end{abstract}

Manuscript submitted April 18, 2016; revised manuscript received May 16, 2016. Published May 26, 2016.

To accelerate an electric vehicle, an important requirement of a battery is the ability to deliver high power pulses at all depths of discharge. ${ }^{1-3}$ Nevertheless, a high power pulse can be difficult to achieve, especially at high depths of discharge (DoD). In some cases, high power is difficult to achieve at high DoD because of an increase in the ohmic resistance during discharge. The ohmic resistance increases due to the movement of the reaction fronts within the electrodes from more favorable (less resistive) to less favorable (more resistive) locations. ${ }^{4,5}$ For instance, this behavior has been documented in the positive electrode of sodium metal-halide batteries, where the low resistivity of the electrode (nickel and/or iron) and the higher resistivity of the electrolyte (sodium tetrachloroaluminate) cause the reaction front to move from the separator to the current collector during discharge. ${ }^{6,7}$ At high $\mathrm{DoD}$, the reaction front is far from the separator and the ionic path length is increased, which increases the overall ohmic resistance in the electrode.

One way to improve the pulse-power performance of an electrode is through the addition of a second active material that only reacts at higher DoD. ${ }^{7,8}$ A schematic of this concept is shown in Figure 1. Part a) shows the discharge and pulse-power process of an electrode with one active material. In this case, the reaction front starts near the separator and moves deeper into the electrode as the cell is discharged. When the electrode is pulsed at the high DoD, the reaction occurs deep within the electrode and there are high ohmic losses due to the increased ionic path length. In contrast, part b) shows the discharge and pulsepower process for an electrode with two active materials. For this case, the same initial behavior is observed, whereby the reaction front moves from the separator to the current collector during discharge. However, during the initial discharge, the second active material does not react. Therefore, when the electrode is pulsed at a high DoD, the high ohmic losses are avoided by reacting the second material close to the separator instead of the first material deep within the electrode. In the figure, the reduction in ionic path length between the two cases (one and two active materials) during the pulse is shown as $\Delta x_{i o n}$. Note that in the second case, the second active material only reacts if the ohmic losses associated with reacting the main active material are greater than the losses associated with the poorer electrochemical performance of the second material.

Researchers have already used the concept outlined in Figure 1 to improve the pulse-power performance of the positive electrode of a sodium metal-halide battery. ${ }^{7,8}$ Galloway and Sudworth both provide descriptions of how the replacement of a small amount of nickel with

\footnotetext{
*Electrochemical Society Student Member.

**Electrochemical Society Member.

${ }^{\mathrm{z} E}$ E-mail: kevin.knehr@columbia.edu
}

iron, which has a lower potential, can improve the pulse power at high DoD. However, these concepts have not been analyzed quantitatively, even though they are strongly supported by data from commercial cells. The aim of this paper is to explore conditions and designs when this concept is viable and if it may be applicable to other battery electrodes.

To accomplish this aim, a generic model was developed based on porous electrode theory, which can simulate the performance of an electrode with two active materials and can be applied to a variety of battery chemistries. Similar models have been developed by other researchers for the positive electrode of a lithium-ion battery. ${ }^{9-12}$ These models were used to study the power and energy characteristics, stresses, and heat generation in electrodes with two active materials, but no analysis of the pulse-power performance was conducted. Furthermore, the concept outlined in Figure 1 is not applicable to the lithium-ion positive electrode because these electrodes discharge uniformly without reaction fronts (see Figure 7 in Ref. 12 for profiles during discharge at a $3 \mathrm{C}$ rate). Therefore, in order to test the viability

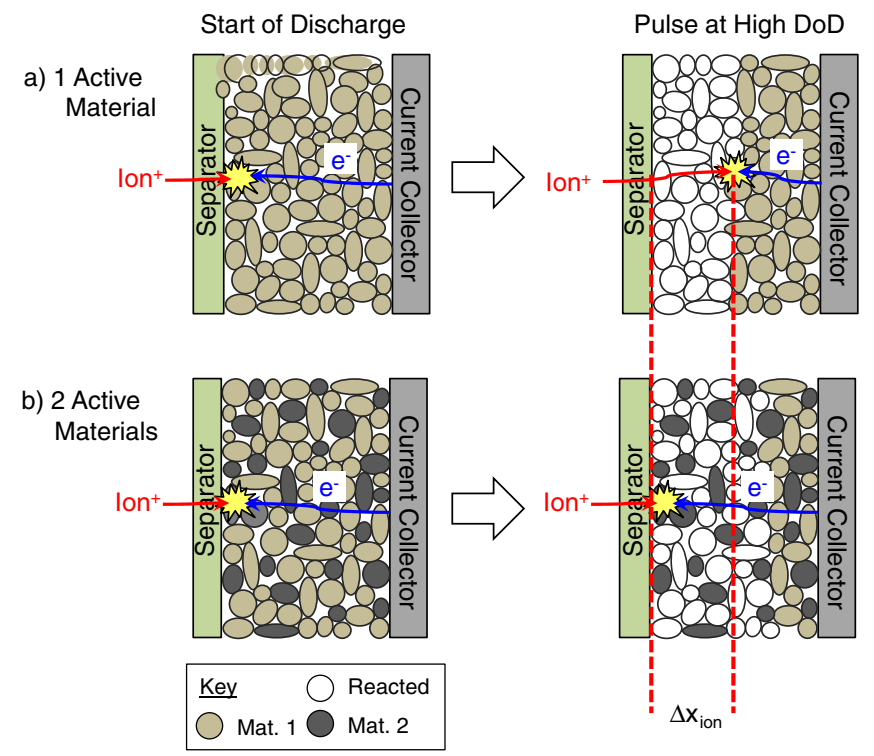

Figure 1. Schematic detailing how the addition of a second electrochemically active material can decrease the ionic resistance within an electrode during pulse-power operation at high depths of discharge. The schematic is valid for electrodes where the ionic resistance is much larger than the ohmic resistance. 
of the concept outlined in Figure 1, different battery chemistries should be analyzed.

In this work, two case studies are conducted on two different electrode chemistries to analyze the viability and applicability of the concept outlined in Figure 1. First, the model is used to conduct an in depth case study on the positive electrode of a sodium metal-halide battery. Next, the analysis is applied to the graphite, negative electrode in a lithium-ion "rocking chair" battery, where it is shown that the addition of other carbonaceous materials to a graphite electrode has the potential to improve the pulse-power performance.

\section{Model Formulation}

Governing equations.-A schematic of the modeling domain, including the dependent variables, is shown in Figure 2. The model is transient, isothermal, and one-dimensional. It is formulated assuming the ohmic resistance in the solid electrode is negligible compared to the ohmic resistance in the electrolyte, i.e.:

$$
\frac{\varepsilon^{1.5} \kappa}{(1-\varepsilon)^{1.5} \sigma} \ll 1
$$

where a Bruggeman-type correction is applied to both conductivities to account for the porous and tortuous nature of the electrode. ${ }^{13}$ This assumption is valid for several commercial electrodes, including the positive electrode of a sodium metal-halide battery and the graphite negative electrode of a lithium-ion battery, which are considered in this work. ${ }^{5,7}$ The validity of Eq. 1 makes it possible to assign a constant value to the potential in the electrode. For numerical simplicity, the potential in the solid electrode is set equal to zero $\left(\phi_{1}=0\right)$. Assuming minimal concentration variations, the potential in the liquid electrolyte is determined using Ohm's law. Conservation of charge, accounting for both faradaic reactions, gives:

$$
\varepsilon^{1.5} \kappa \frac{\partial^{2} \phi_{2}}{\partial x^{2}}=-a_{I} \varepsilon_{I, 0} i_{r x n, I}-a_{I I} \varepsilon_{I I, 0} i_{r x n, I I}
$$

In this model, the specific surface area, $a$, is an intrinsic property of the electrochemically active material. It is defined as the surface area of the active material per volume of the active material. This definition differs from that of Newman, where the specific surface area is defined as the surface area of the material per unit volume of the electrode. ${ }^{14}$ In Eq. 2, $\varepsilon$ is the volume fraction of the liquid electrolyte within the electrode and $\varepsilon_{I, 0}$ and $\varepsilon_{I I, 0}$ are the initial volume fractions of the $1^{\text {st }}$ and $2^{\text {nd }}$ electrochemically active materials in the electrode. All three values are assumed to be independent of DoD.

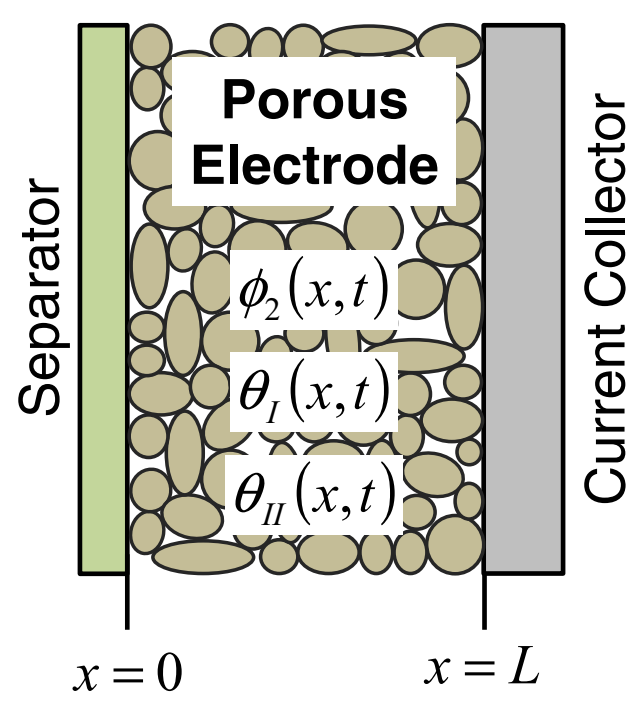

Figure 2. Schematic of the modeling domain and variables used in the pulsepower simulations.
The reaction currents $\left(i_{r x n}\right)$ are determined assuming the active materials undergo the following reaction:

$$
A_{k} \leftrightarrow A_{k}^{n_{k}+}+\mathrm{n}_{k} \mathrm{e}^{-}
$$

where the subscript $k$ denotes active material $I$ or $I I$. This work is focused on simulating the maximum power of an electrode, which is obtained at high applied currents. At these high currents, the reaction kinetics are modeled by assuming the following Tafel equation:

$$
i_{r x n, k}=i_{0} \theta_{k} \gamma \exp \left[\frac{\gamma \alpha F}{R T}\left(-\phi_{2}-U_{k}\right)\right]
$$

where it is assumed the charge transfer coefficient, $\alpha$, has the same value for both active materials. In Eq. 4, the open circuit potential $\left(U_{k}\right)$ is set equal to the standard reference potential due to the assumption of negligible concentration variations in the electrolyte. In addition, $\gamma$ is defined as:

$$
\gamma=\frac{i_{a p p}}{\left|i_{a p p}\right|}
$$

The inclusion of $\gamma$ generalizes the model formulation and makes it possible to simulate the cathodic and anodic discharge of an electrode.

The kinetic rate equation (Eq. 4) assumes that the reaction is directly proportional to $\theta_{k}$, which is the ratio of the volumetric discharge capacity of the material to the initial volumetric discharge capacity of the material, where the volume is defined with respect to the electrode. $\theta_{k}$ is defined as:

$$
\theta_{k}=\frac{\hat{Q}_{k}}{\hat{Q}_{k, 0}} ; \quad \hat{Q}_{k, 0}=\frac{\varepsilon_{k, 0} n_{k} F \rho_{k}}{M_{k}}
$$

For an electrode undergoing a conversion reaction, the change in $\theta_{k}$ corresponds to a reduction in the volume of the active material. For an electrode undergoing an intercalation reaction, the change in $\theta_{k}$ corresponds to a reduction in the available sites for intercalation. The change in $\theta_{k}$ is simulated using the following:

$$
\frac{\partial \theta_{k}}{\partial t}=-\frac{\gamma a_{k} \varepsilon_{k, 0} i_{r x n, k}}{\hat{Q}_{k, 0}}
$$

Boundary and initial conditions.-The simulations assume controlled-current operation of the electrode. The boundary conditions for the electrolyte potential $\left(\phi_{2}\right)$ are set using Ohm's law. At the separator, the current density is set equal to the applied current density, i.e.:

$$
\left.\varepsilon^{1.5} \kappa \frac{\partial \phi_{2}}{\partial x}\right|_{x=0}=i_{a p p}
$$

At the current collector, the ionic current density is set equal to zero, as follows:

$$
\left.\frac{\partial \phi_{2}}{\partial x}\right|_{x=L}=0
$$

At the start of the simulation, the potential difference between the electrode and electrolyte is set equal to the open circuit potential of the $1^{\text {st }}$ active material, which results in the following:

$$
\left.\left(\phi_{1}-\phi_{2}\right)\right|_{t=0}=U_{I} \text { or }\left.\phi_{2}\right|_{t=0}=-U_{I}
$$

In addition, $\theta_{k}$ is set using the following initial conditions:

$$
\left.\theta_{k}\right|_{t=0}=1
$$

Dimensionless formulation.-This section introduces a set of three scaled variables, which are used to reformulate the governing equations (Eqs. 2, 4, 6-11), from which three dimensionless numbers emerge. The three scaled variables are defined as:

$$
\bar{\phi}_{2}=\frac{\varepsilon^{1.5} \kappa}{\left|i_{\text {base }}\right| L} \phi_{2} ; \quad \bar{x}=\frac{x}{L} ; \quad \tau=\frac{\left|i_{\text {base }}\right|}{\left(\hat{Q}_{I, 0}+\hat{Q}_{I I, 0}\right) L} t
$$


where $i_{\text {base }}$ is the baseline current density applied before the high current pulse (see discussion of Figure 3 for more detail). These variables are used to reformulate Eqs. 2, 4, 6-7, which results in the following, dimensionless governing equations:

$$
\begin{gathered}
\frac{\partial^{2} \bar{\phi}_{2}}{\partial \bar{x}^{2}}=-\gamma \psi\left(\varepsilon_{I, 0} \theta_{I}+\xi \varepsilon_{I I, 0} \theta_{I I}\right) \exp \left(-\gamma w_{T}^{-1} \bar{\phi}_{2}\right) \\
\frac{\partial \theta_{I}}{\partial \tau}=-\psi \varepsilon_{I, 0} \theta_{I} f_{I, 0}^{-1} \exp \left(-\gamma w_{T}^{-1} \bar{\phi}_{2}\right) \\
\frac{\partial \theta_{I I}}{\partial \tau}=-\xi \psi \varepsilon_{I I, 0} \theta_{I I} f_{I I, 0}^{-1} \exp \left(-\gamma w_{T}^{-1} \bar{\phi}_{2}\right)
\end{gathered}
$$

where $f_{k, 0}$ is the initial capacity fraction of the active material in the electrode defined as follows:

$$
f_{k, 0}=\frac{\hat{Q}_{k, 0}}{\hat{Q}_{I, 0}+\hat{Q}_{I I, 0}}
$$

In Eqs. 13-15, the dimensionless constants are defined as:

$$
\begin{gathered}
w_{T}=\frac{\varepsilon^{1.5} \kappa R T}{\alpha F\left|i_{\text {base }}\right| L} \\
\xi=\frac{a_{I I} i_{0, I I}}{a_{I} i_{0, I}} \exp \left(-\frac{\gamma \alpha F\left(U_{I I}-U_{I}\right)}{R T}\right) \\
\psi=\frac{a_{I} i_{0, I} L}{\left|i_{\text {base }}\right|} \exp \left(-\frac{\gamma \alpha F U_{I}}{R T}\right)
\end{gathered}
$$

$w_{T}$ is the ratio of charge transfer to ohmic resistance, ${ }^{15} \xi$ is the ratio of kinetic and thermodynamic parameters between active materials I and II, and $\psi$ is a dimensionless description of the kinetic and thermodynamic parameters of active material I. The value of $\psi$ has an impact on the absolute power obtainable for a system, but it does not influence whether or not the addition of active material II will enhance the pulse-power performance.

As a result of the reformulation, the boundary conditions and initial conditions on the electrolyte potential (Eqs. 8-10) are redefined as:

$$
\begin{gathered}
\left.\frac{\partial \bar{\phi}_{2}}{\partial x}\right|_{x=0}=\frac{i_{\text {app }}}{\left|i_{\text {base }}\right|} ;\left.\quad \frac{\partial \bar{\phi}_{2}}{\partial x}\right|_{x=L}=0 \\
\left.\bar{\phi}_{2}\right|_{t=0}=-\frac{U_{I} \varepsilon^{1.5} \kappa}{\left|i_{\text {base }}\right| L}
\end{gathered}
$$

The initial conditions for the volume fractions of the active materials (Eq. 11) are unchanged by the introduction of the scaled variables.

Numerical methods.-Equations 13-21 were linearized and solved simultaneously in FORTRAN 95 using the subroutines BANDJ and MATINV. ${ }^{14}$ Discretization of the equations was done using the forward time, central difference method. A mesh size of 201 points and a time step of 0.01 seconds were used. Computer experiments were performed for the mesh size and time step in order to test for convergence. The computing was performed on the Yeti Shared HPC Cluster at Columbia University.

Description of model system.-The positive electrode of a sodiummetal halide battery was selected as a test case for the pulse-power analysis. In a commercial sodium metal-halide battery, nickel and iron are used as the first and second electrochemically active materials, respectively. During discharge, the following half-cell reactions occur:

$$
\begin{aligned}
& \mathrm{Ni}^{2+}+2 \mathrm{e}^{-} \rightarrow \mathrm{Ni} \quad U_{I}=2.58 \mathrm{~V} \\
& \mathrm{Fe}^{2+}+2 \mathrm{e}^{-} \rightarrow \mathrm{Fe} \quad U_{I I}=2.34 \mathrm{~V}
\end{aligned}
$$

Table I. List of parameters used in case study of the positive electrode in a sodium metal-halide battery.

\begin{tabular}{llc} 
Symbol & Description & Value \\
\hline$a_{I}, a_{I I}$ & Specific surface area $\left(\mathrm{cm}^{2} \mathrm{~cm}^{-3}\right)$ & 45.5 \\
$\alpha$ & Charge transfer coefficient & 0.5 \\
$\varepsilon$ & Porosity & 0.5 \\
$i_{\text {base }}$ & Baseline discharge current density $\left(\mathrm{A} \mathrm{cm}^{-2}\right)^{16}$ & $1.59 \times 10^{-1}$ \\
$i_{0, I}, i_{0, I I}$ & Exchange current density $\left(\mathrm{A} \mathrm{cm}^{-2}\right)$ & $1.02 \times 10^{-2}$ \\
$\kappa$ & Electrolyte conductivity $\left(\mathrm{S} \mathrm{cm}^{-1}\right)^{27}$ & 0.778 \\
$T$ & Temperature $(\mathrm{K})$ & 573
\end{tabular}

were the open circuit potentials are given with respect to the $\mathrm{Na} \mid \mathrm{Na}^{+}$ negative electrode of the sodium metal-halide battery. In the positive electrode, the iron and nickel ions exist as sparingly soluble metal chlorides with the remainder of the solid phase comprised of nickel metal as a conductive backbone. The initial volume fractions of nickel chloride and iron chloride $\left(\varepsilon_{I, 0}\right.$ and $\varepsilon_{I I, 0}$, respectively) were determined assuming a constant volumetric capacity of $1777 \mathrm{C} \mathrm{cm}^{-3}$ for the electrode. In a commercial battery, the electrode is assembled in the discharged state as nickel and iron metal with sparingly soluble sodium chloride determining the total capacity. In this study, the constant volumetric capacity of $1777 \mathrm{C} \mathrm{cm}^{-3}$ corresponds to a fully discharged electrode with a composition of 61-65\% (by weight) nickel and iron metal and 35-39\% (by weight) sodium chloride, where the exact weight fractions depend on the initial capacity fractions of the two active materials $\left(f_{I, 0}\right.$ and $\left.f_{I I, 0}\right)$. These values agree well with the compositions studied by Zhu et al. ${ }^{16}$

Table I provides the values of all other parameters used in the simulations. The specific surface area was calculated using the following equation:

$$
a_{k}=\frac{3}{r_{p, k}}
$$

with an average particle radius $\left(r_{p, k}\right)$ of $660 \mu \mathrm{m}$ for both materials. ${ }^{16}$ Note that this electrode satisfies the assumption outlined in Eq. 1 that the ohmic resistance in the solid electrode is negligible compared to the ohmic resistance in the electrolyte. For instance, evaluation of Eq. 1 with the parameters in Table I (and a conductivity of $3.92 \times 10^{4} \mathrm{~S} \mathrm{~cm}^{-1}$ for the solid nickel electrode) results in a value of $2.0 \times 10^{-5} \ll 1 .^{17}$ In addition, the assumption of minimal concentration variations in the electrolyte is valid for this electrode because the reactants exist as sparingly soluble chlorides, which are evenly dispersed throughout the electrode.

Simulation procedure.-This section contains a description of the simulation procedure that was employed to analyze the pulse-power performance of an electrode. Figure 3 shows an example of a simulated discharge curve for a sodium metal-halide electrode containing no iron (i.e., $f_{I I, 0}=0$ ). First, the electrode was discharged galvanostatically at a current $\left(i_{\text {base }}\right)$ to a set depth of discharge (DoD). Once the DoD was reached, a high current pulse $\left(i_{\text {pulse }}\right)$ was applied to the electrode for a time $\left(t_{\text {pulse }}\right)$. For both case studies, $t_{\text {pulse }}$ is equal to 10 seconds, which was chosen based on the discharge specifications set by the U. S. Advanced Battery Consortium (USABC) for plug-in hybrid electric vehicles. ${ }^{3}$ The voltage at the end of the high current pulse is used to determine the pulse power of the electrode $\left(P_{\text {pulse }}\right)$, which is the basis for the analysis below. To determine the voltage, the potential drop across the electrode was considered relative to the open circuit potential of a Na|Na+ electrode as follows:

$$
V_{\text {cell }}=\left(\left.\phi_{1}\right|_{x=L}-\left.\phi_{2}\right|_{x=0}\right)-U_{N a \mid N a^{+}}=-\left.\phi_{2}\right|_{x=0}
$$

where the open circuit potential is equal to zero $\left(U_{\mathrm{Na}_{\mathrm{Na}}+}=0\right)$ because all open circuit potentials are taken with respect to the $\mathrm{Na} \mid \mathrm{Na}^{+}$ electrode in this case study. 


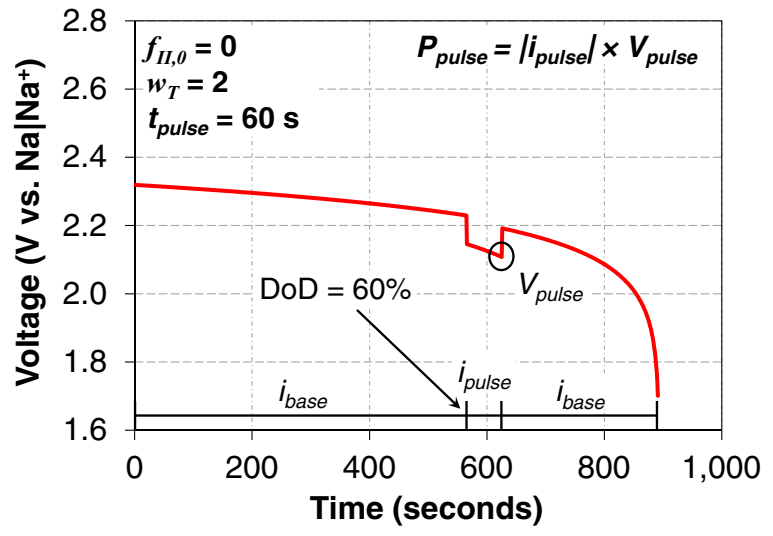

Figure 3. Simulated discharge curve exemplifying procedure used to analyze the pulse-power performance of the electrode.

\section{Results and Discussion}

Power curves for the metal-halide electrode.-Figures 4-6 compare the $P_{\text {pulse }}$ of electrodes containing one active material to the $P_{\text {pulse }}$ of electrodes containing two active materials, where all electrodes have the same volumetric capacity $\left(\mathrm{C} \mathrm{cm}^{-3}\right)$. In Figure 4, the second electrochemically active material (iron chloride) accounted for $1 \%$ of the total capacity. In Figure 5, the iron chloride accounted for $10 \%$ of the capacity. In Figure 6 , it accounted for $50 \%$ of the capacity. For all active material fractions, simulations were run to DoDs of $60 \%$ $(\tau=0.6)$ and $80 \%(\tau=0.8)$ for electrodes with three different values
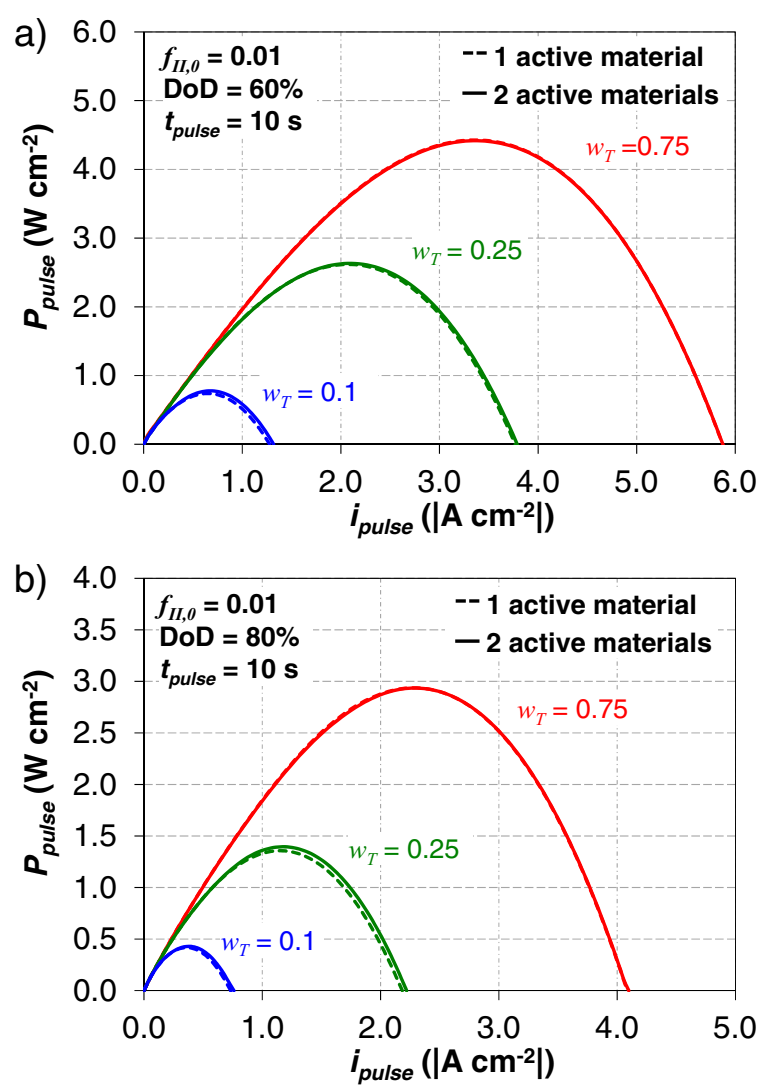

Figure 4. Comparison of simulated pulse-power $\left(P_{\text {pulse }}\right)$ vs. pulse-current $\left(i_{\text {pulse }}\right)$ for electrodes comprised of one and two active materials that were discharged to a DoD of a) $60 \%(\tau=0.6)$ and b) $80 \%(\tau=0.8)$. For the two-material simulations, the second material accounted for $1 \%$ of the total capacity. Definitions for $P_{\text {pulse }}$ and $i_{\text {pulse }}$ can be found on Figure 3.
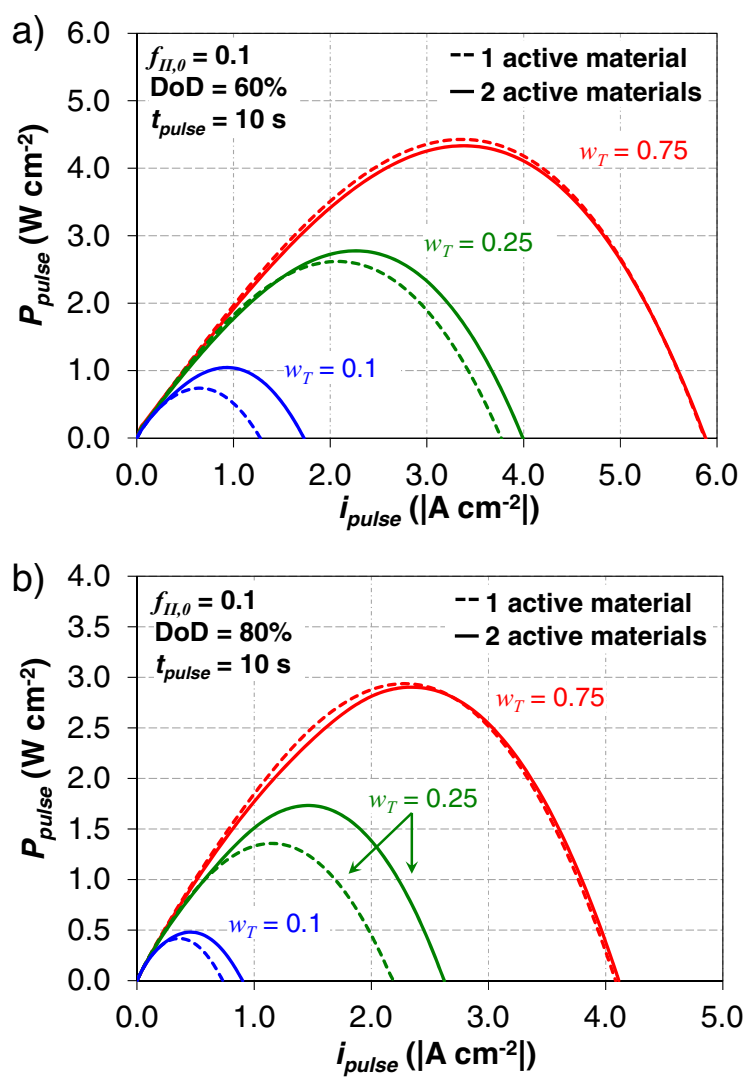

Figure 5. Comparison of simulated pulse-power $\left(P_{\text {pulse }}\right)$ vs. pulse-current $\left(i_{\text {pulse }}\right)$ for electrodes comprised of one and two active materials that were discharged to a DoD of a) $60 \%(\tau=0.6)$ and b) $80 \%(\tau=0.8)$. For the two-material simulations, the second material accounted for $10 \%$ of the total capacity. Definitions for $P_{\text {pulse }}$ and $i_{\text {pulse }}$ can be found on Figure 3.

of $w_{T}(0.1,0.25$, and 0.75$)$. The values of $w_{T}$ in the simulations were adjusted by changing the length of the electrode. For each value of $w_{T}$, the electrodes with one and two active materials had the same total capacity. To put $w_{T}$ into perspective, note that for the commercial sodium metal-halide cell published by Rijssenbeek et al., a discharge at $\mathrm{C} / 3$ (10 amps) would correspond to $w_{T} \sim 0.64 .^{6}$ This value was determined using the parameters in Table I along with an average path length of $1 \mathrm{~cm}$ and an electrode area of $236 \mathrm{~cm}^{2}$. The path length and electrode area were obtained through an ImageJ analysis of Figure 1 in Ref. 6.

Figure 4 shows that replacement of $1 \%$ of the capacity with the second electrochemically active material has a negligible impact on the pulse-power of the electrode. For both pulse-start times (i.e., DoDs) and all three values of $w_{T}$, the $P_{\text {pulse }}$ vs. $i_{\text {pulse }}$ curves are almost identical for the simulations with 1 and 2 active materials. Replacement of $1 \%$ of the capacity has no impact on the pulse-power because the second material does not have enough capacity to maintain the reaction front near the separator during the high current pulse. Instead, the electrode behaves like there is only one active material and large ohmic losses are still present.

In contrast, Figure 5 shows that the replacement of $10 \%$ of the capacity with a second active material can improve the pulse-power performance. For both DoDs and for $w_{T}$ equal to 0.1 and 0.25 , the electrodes with two active materials have a higher maximum pulse power than the electrodes with one active material. For $w_{T}=0.1$, the second material increases the maximum power by $41 \%$ and $15 \%$ at DoDs of $60 \%(\tau=0.6)$ and $80 \%(\tau=0.8)$, respectively. For $w_{T}=0.25$, the second material increases the maximum power by $6 \%$ and $28 \%$ at DoDs of $60 \%$ and $80 \%$, respectively. In contrast, the second material is shown to slightly decrease $(\sim 2 \%)$ the pulse power 

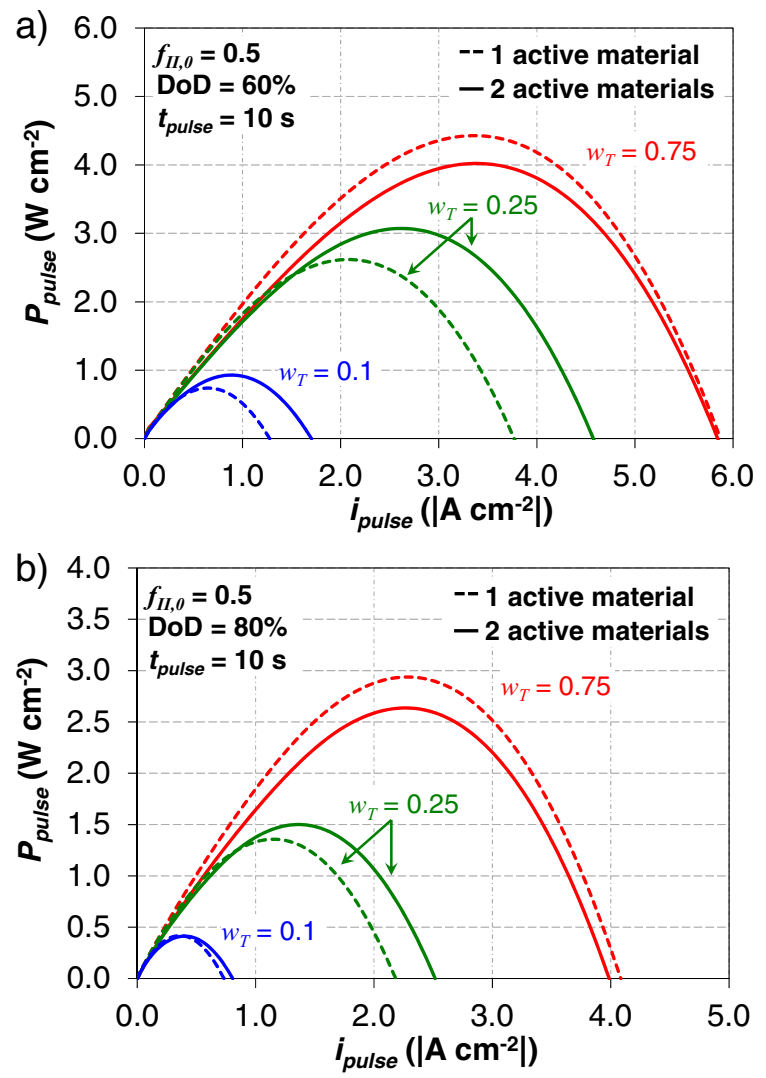

Figure 6. Comparison of simulated pulse-power $\left(P_{\text {pulse }}\right)$ vs. pulse-current $\left(i_{\text {pulse }}\right)$ for electrodes comprised of one and two active materials that were discharged to a DoD of a) 60\% ( $\tau=0.6)$ and b) $80 \%(\tau=0.8)$. For the two-material simulations, the second material accounted for $50 \%$ of the total capacity. Definitions for $P_{\text {pulse }}$ and $i_{\text {pulse }}$ can be found on Figure 3.

for $w_{T}=0.75$. The higher $w_{T}$ corresponds to a more uniform reaction distribution during the initial, baseline discharge. ${ }^{15}$ This suggests that for higher values of $w_{T}$ the first active material is still present close to the separator when the high current pulse is applied. Therefore, there is no benefit for adding the second active material.

Figure 6 shows that replacement of $50 \%$ of the capacity with the second active material has a similar trend to replacement of $10 \%$ of the capacity. For $w_{T}=0.1$, the second material increases the maximum power by $26 \%$ and $<1 \%$ at DoDs of $60 \%$ and $80 \%$, respectively. For $w_{T}=0.25$, the second material increases the maximum power by $17 \%$ and $11 \%$ at DoDs of $60 \%$ and $80 \%$, respectively. In addition, for $w_{T}=0.75$ the second material decreases the maximum power by $10 \%$ and $11 \%$ at DoDs of $60 \%$ and $80 \%$, respectively. The decrease in power is once again due to the uniform reaction distribution during the initial baseline discharge. For both electrodes with $w_{T}=0.75$ (with and without the second active material), the reaction occurs close to the separator during the high current pulse. However, for the 50/50 composition of the active materials, almost all of the first active material has been consumed during the baseline discharge to $60 \%$ and $80 \%$ DoD. Therefore, the relatively poor pulse-power of this electrode is caused by the poor electrochemical performance of the second active material.

In summary, Figures 4 to 6 demonstrate that the addition of a second electrochemically active material to an electrode can significantly improve the pulse power. The level of improvement depends on the amount of second active material as well as the electrode depth of discharge. Additionally, significant improvements were only observed for electrodes with low $w_{T}(<0.75)$, due to the uneven reaction distributions during the baseline discharge, which deplete the active material near the separator.
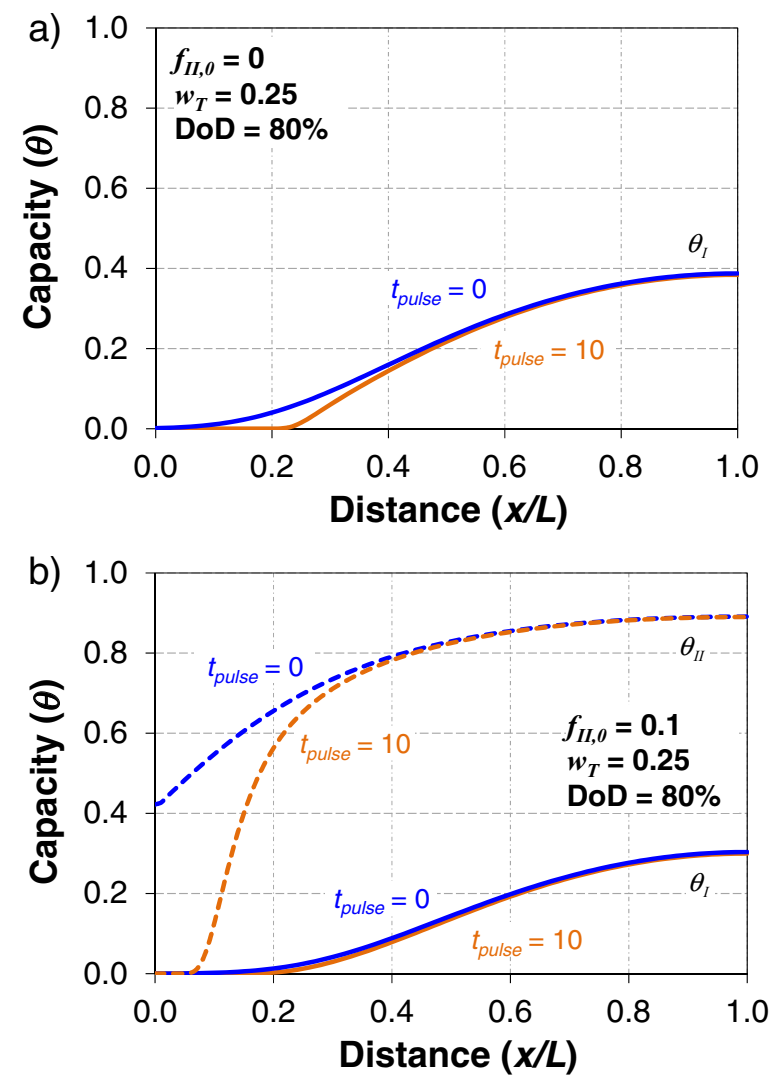

Figure 7. Simulated distributions of active materials during a high current pulse at $i_{\text {pulse }}=10 \times i_{\text {base }}$ for a) an electrode with only the first active material and b) an electrode with $10 \%$ of the capacity from the second active material. $x / L=0$ and $x / L=1$ correspond to the separator and current collector, respectively.

While the addition of a second electrochemically active material can improve the pulse power of the electrode, it can also decrease the theoretical energy density. For instance, replacement of $1 \%, 10 \%$, and $50 \%$ of the capacity with the second active material decreases the theoretical energy density of the electrode by $0.1 \%, 0.9 \%$, and $4.7 \%$, respectively. This decrease in energy is due to the lower voltage of the second material (iron chloride). Although the decrease in energy density is slight, the tradeoff between increasing power and energy remains an important consideration for designing an electrode with two active materials.

Active material distributions. - The objective of this section is to show that the increase in pulse power for the electrodes with two active materials is due to the mechanism outlined in the introduction (see Figure 1). Figure 7 shows the simulated distributions of active material within a) an electrode comprised of only the first active material and b) an electrode where the second active material accounts for $10 \%$ of the capacity. The distributions are shown during a high current pulse at $i_{\text {pulse }}=10 \times i_{\text {base }}$.

Fig. 7 a shows that when only one active material is present, the high current pulse results in the quick consumption of the active material near the separator, which forces the reaction front into the electrode. At the end of the pulse, all of the material within the first $20 \%$ of the electrode closest to the separator is consumed. In contrast, for the electrode with two active materials (Fig. 7b), the high current pulse has a minimal impact on the distribution of the first active material. Instead, only the second active material is consumed. However, because there was a large amount of the second active material near the separator at the start of the pulse, the subsequent reactions only consumed the material within the first $10 \%$ of the electrode closest 


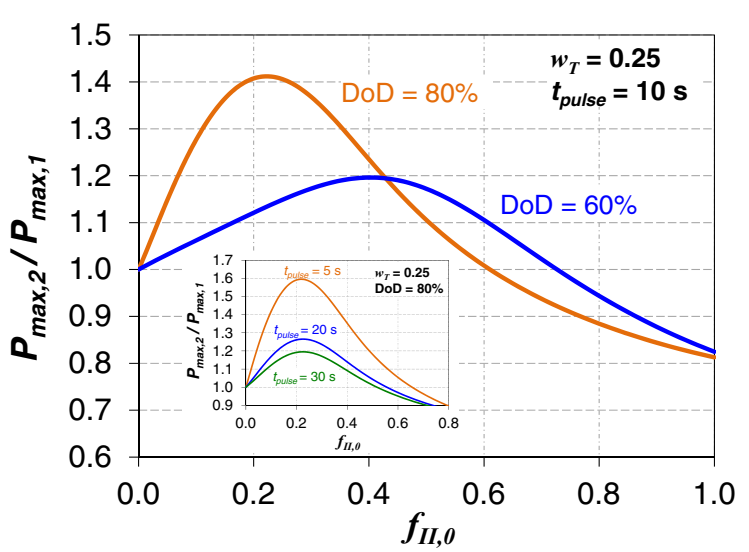

Figure 8. Simulated pulse-power as a function of the capacity fraction of the second active material (iron chloride) in the positive electrode of a sodium metal-halide battery. Pulse-power is plotted as the ratio of the maximum pulsepower of an electrode with two active materials (nickel + iron chloride) to the maximum pulse-power of an electrode with one active material (nickel chloride). Inset: pulse-power for pulse times of 5, 20, and 30 seconds.

to the separator. Therefore, at the end of the pulse, the electrode with two active materials has only half as much ohmic resistance as the electrode with one active material (10\% vs. $20 \%$ reaction penetration depth). This resulted in an overall improvement in the pulse-power performance (see Figure 5b).

Electrode design considerations.-The results in Figures 4 to 6 indicate that the pulse-power performance of an electrode depends on the fractions of the first and second electrochemically active materials. This suggests that the model can be used as a design tool to determine what active material fractions maximize the pulse power of an electrode. The results of such an analysis for the sodium metal-halide electrode are shown in Figure 8 for an electrode with $w_{T}=0.25$ that underwent high current pulses for 10 seconds. Two curves are shown for pulses after baseline discharges to $60 \%$ and $80 \% \mathrm{DoD}$. The curves show how the maximum pulse power of the electrode changes as a function of the capacity fraction of the second electrochemically active material. The power is plotted as the ratio of the maximum pulse power for an electrode with two active materials to the maximum pulse power of an electrode with one active material. Values greater than one indicate improvement in performance with the addition of the second material. The maximum pulse power was obtained from the peak of $P_{\text {pulse }}$ vs. $i_{\text {pulse }}$ curves (see Figures 4 to 6 for examples).

The two, main curves in Figure 8 indicate that the optimal capacity fraction of the second material depends on the DoD of the electrode. Under the current operating conditions, the simulations suggest that electrodes with capacity fractions $\left(f_{I I, 0}\right)$ of 0.2 to 0.5 can significantly improve the pulse power at both depths of discharge (up to $20 \%$ and $40 \%$ improvement for DoD equal to $60 \%$ and $80 \%$, respectively). Note that electrodes with capacity fractions in this range will also have $1.9 \%$ to $4.7 \%$ lower theoretical energy densities than an electrode with only the first active material. For the positive electrode of a sodium metalhalide cell, this range corresponds to a composition in the discharged state of $\sim 3 \%$ to $\sim 9 \%$ (by weight) iron, $\sim 35 \%$ sodium chloride, and the remainder nickel.

The inset in Figure 8 shows results for pulses of 5, 20, and 30 seconds for pulses at $80 \%$ DoD. The results indicate that increases in the pulse time $\left(t_{\text {pulse }}\right)$ slightly decrease both the range of beneficial capacity fractions and the net improvement in pulse power. This trend is expected since higher pulse times will consume more of the second active material and push the reaction front deeper into the electrode.

In addition to helping determine the best active material fractions for an electrode, the model can also be used to analyze which materials would be most beneficial as a second active material. The results of such an analysis are shown in Figure 9 for electrodes that underwent

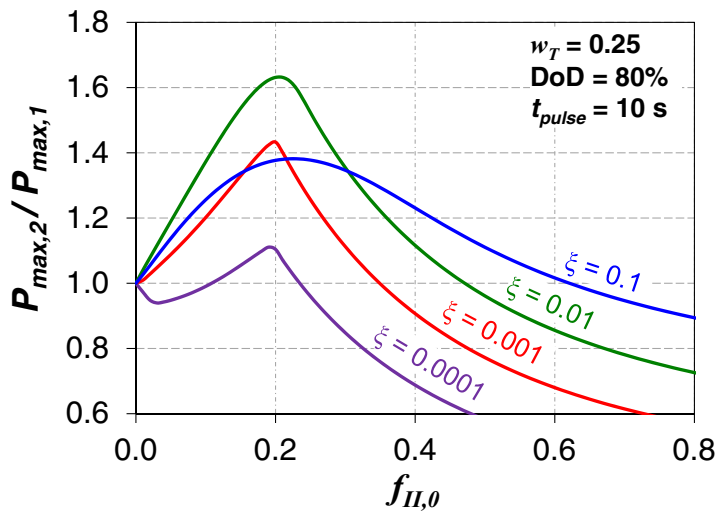

Figure 9. Comparison of the simulated pulse-power for electrodes with different values of $\xi$ (Eq. 18), which corresponds to different electrochemical properties for the second active material. Pulse-power is plotted as the ratio of the maximum pulse-power of an electrode with two active materials to the maximum pulse-power of an electrode with one active material.

high current pulses for 10 seconds at $80 \%$ DoD. All simulations were conducted for electrodes with the same geometry and the same first active material, which corresponded to constant values of $w_{T}=0.25$ and $\psi=4.4 \times 10^{11}$ (Eqs. 17 and 19). The only difference between each curve is the value of $\xi$ used in the simulations, which depends on the electrochemical properties of the second active material (Eq. 18). $\xi=1$ corresponds to identical first and second electrochemically active materials. Note that $\xi=0.088$ for the sodium metal-halide electrode used in the case study (Figures 4 to 8 ).

Figure 9 shows how the pulse power of the electrode changes with respect to changes in the capacity fraction of the second active material $\left(f_{I I, 0}\right)$ and the value of $\xi$. The power ratios in Figure 9 have the same meaning as the power ratios in Figure 8 . The results of the simulations predict that the improvement in pulse power will go through a maximum as the value of $\xi$ increases. For instance, the peak pulse power $\left(P_{\text {peak }}(\xi)\right)$ follows the trend $P_{\text {peak }}(0.01)>P_{\text {peak }}(0.001)>P_{\text {peak }}(0.1)$ $>P_{\text {peak }}(0.0001)$. At low values of $\xi$, the electrochemical properties of the second material are too poor to provide any benefit during pulse power. This is exemplified for $\xi=0.0001$, where simulations predict only a $<12 \%$ increase in pulse power can be achieved for a small range of capacity fractions ( 0.11 to 0.23$)$. In contrast, at high values of $\xi$, the electrochemical properties of the second active material become too similar to the first, which causes the second material to react during the baseline discharge. This reaction will move the reaction front of the second material away from the separator, which increases the ohmic drop during a high current pulse and decreases the power. This is exemplified by the decrease in the maximum pulse power from $\xi=0.01$ to $\xi=0.1$.

Negative electrode of a Li-ion battery.-In the previous sections, the sodium metal-halide electrode was used as a test case to analyze how the addition of a second electrochemically active material can improve the pulse power of an electrode. In this section, a second, brief case study is conducted to demonstrate how the addition of other carbonaceous materials (in particular, carbon black) to the graphite electrode of a commercial lithium-ion battery may also improve the pulse power.

To accomplish this, the model outlined in Equations 11, 13-21 is utilized with adjustments made to the input parameters. The input parameters for the Li-ion case study are provided in Table II. The geometric parameters for graphite (the first active material) were obtained from an analysis of a commercial 18500 graphite electrode using Xray nano-tomography recently published by Ender. ${ }^{18}$ The exchange current density $\left(i_{0, I}\right)$ was calculated using Eq. 7 in Ref. 19 assuming a lithium salt concentration of $1 \mathrm{M}$ and a $50 / 50$ fraction of filled to unfilled intercalation sites. For carbon black (the second active material), the specific surface area was calculated using Eq. 24 assuming 


\begin{tabular}{|c|c|c|}
\hline Symbol & Description & Value \\
\hline$a_{I}$ & $\begin{array}{l}\text { Specific surface area of graphite } \\
\left(\mathrm{cm}^{2} \mathrm{~cm}^{-3}\right)^{18}\end{array}$ & $3.6 \times 10^{3}$ \\
\hline$a_{I I}$ & $\begin{array}{l}\text { Specific surface area of carbon black } \\
\left(\mathrm{cm}^{2} \mathrm{~cm}^{-3}\right)^{20}\end{array}$ & $1.2 \times 10^{6}$ \\
\hline$\alpha$ & Charge transfer coefficient & 0.5 \\
\hline$\varepsilon$ & Porosity ${ }^{18}$ & 0.182 \\
\hline$\kappa$ & Electrolyte conductivity $\left(\mathrm{S} \mathrm{cm}^{-1}\right)^{19}$ & $9.48 \times 10^{-3}$ \\
\hline$i_{0, I}$ & $\begin{array}{l}\text { Exchange current density of graphite } \\
\left(\mathrm{A} \mathrm{cm}^{-2}\right)(\text { Eq. } 7 \text { in Ref. 19) }\end{array}$ & $2.8 \times 10^{-4}$ \\
\hline$i_{0, I I}$ & $\begin{array}{l}\text { Exchange current density of carbon } \\
\text { black }\left(\mathrm{A} \mathrm{cm}^{-2}\right)^{21}\end{array}$ & $4.1 \times 10^{-5}$ \\
\hline$L$ & Electrode thickness $(\mathrm{cm})^{18}$ & 0.0076 \\
\hline$T$ & Temperature (K) & 298 \\
\hline$\tau_{\text {tort }}$ & Tortuosity ${ }^{18}$ & 11.2 \\
\hline$U_{I}$ & $\begin{array}{l}\text { Average open circuit potential of } \\
\text { graphite (V) }\end{array}$ & 0.1 \\
\hline$U_{I I}$ & $\begin{array}{l}\text { Average open circuit potential of } \\
\text { carbon black }(\mathrm{V})\end{array}$ & 0.5 \\
\hline
\end{tabular}

a particle diameter of $50 \mathrm{~nm} .{ }^{20}$ The exchange current density of carbon black reflects the most conservative estimate for a carbonaceous material (petroleum coke) that could be found in the literature. ${ }^{21}$

For both carbon materials, discharging of the electrode results in the oxidation of intercalated lithium, which can be expressed as follows:

$$
\mathrm{Li} \rightarrow \mathrm{Li}^{+}+\mathrm{e}^{-}
$$

Both carbon materials undergo this reaction; however, each material has a different set of kinetic and thermodynamic parameters. It is assumed both materials contain a maximum of one lithium per six carbon $\left(\mathrm{LiC}_{6}\right)$ at the start of discharge. ${ }^{22}$ The open circuit potentials for the reactions ( $U_{I}$ and $U_{I I}$ in Table II) were determined from data in the literature. ${ }^{19,20,22,23}$ Both are given with respect to a $\mathrm{Li} \mid \mathrm{Li}^{+}$electrode and correspond to the average open circuit potential of the reaction. This simplification to the open circuit potential is necessary for incorporation into the simple model. A full treatment, which is out of the scope of this work, would incorporate variations in the open circuit potential with degree of lithiation.

The only change in the model description for the Li-ion case study is the use of Eq. 16 in Reference 19 to define the effective conductivity instead of a Bruggeman correction. This corresponds to replacing $\varepsilon^{1.5}$ with $\varepsilon / \tau_{\text {tort }}$ everywhere in the model formulation (Equations 1, 17 and $21)$. Note that with or without this change, the electrode still satisfies the condition outlined in Eq. 1 that the ohmic resistance in the solid electrode is negligible. For instance, evaluation of Eq. 1 with and without this change using the parameters in Table II (and a value of $(1-\varepsilon)^{1.5} \sigma=10 \mathrm{~S} \mathrm{~cm}^{-1}$ for the solid carbon electrode) results in values of $2.0 \times 10^{-4} \ll 1$ and $1.0 \times 10^{-3} \ll 1$, respectively. ${ }^{19}$ To determine the battery voltage, the potential drop across the carbon electrode was considered relative to the open circuit potential of a $\mathrm{LiCoO}_{2} \mid \mathrm{CoO}_{2}$ electrode as follows:

$$
V_{\text {cell }}=U_{\mathrm{LiCoO}_{2}}-\left(\left.\phi_{1}\right|_{x=L}-\left.\phi_{2}\right|_{x=0}\right)=U_{\mathrm{LiCoO}_{2}}+\left.\phi_{2}\right|_{x=0}
$$

where $U_{\mathrm{LiCoO}_{2}}$ is taken with respect to a $\mathrm{Li} \mid \mathrm{Li}^{+}$electrode $(3.8 \mathrm{~V})$.

Figure 10 shows the results for simulations of a graphite electrode that underwent 10 second pulses after a baseline discharge at $1 \mathrm{C}$ and $2 \mathrm{C}$ to $80 \%$ DoD. All simulated electrodes have the same volumetric capacity. The figure plots the change in pulse power due to changes in the capacity fraction of carbon black. The pulse power is plotted as the ratio of the maximum pulse power of an electrode with two active materials (carbon black + graphite) to the maximum pulse power of an electrode with one active material (graphite). The results indicate that addition of carbon black to the negative electrode could improve the pulse-power performance of a Li-ion battery. For instance, the

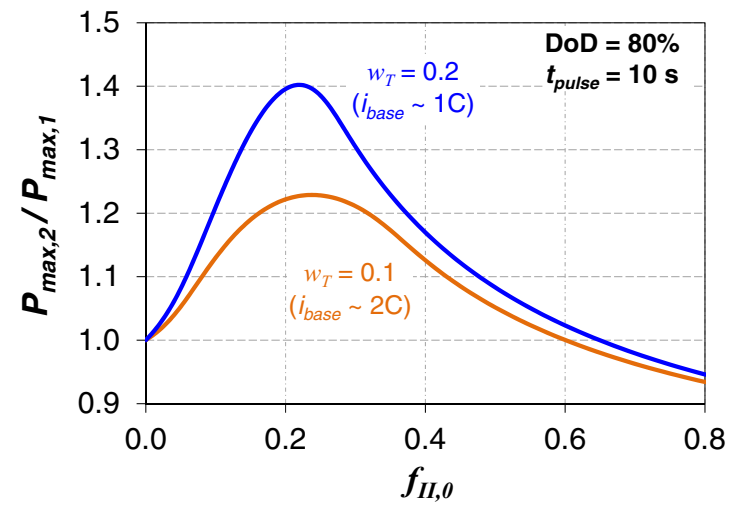

Figure 10. Simulated pulse-power as a function of the capacity fraction of the second active material (carbon black) in the negative electrode of a Li-ion battery. Pulse-power is plotted as the ratio of the maximum pulse-power of an electrode with two active materials (carbon black + graphite) to the maximum pulse-power of an electrode with one active material (graphite).

simulations in Figure 10 show that replacement of $0-60 \%$ of the graphite capacity with carbon black will increase the pulse power at both discharge rates. For the $1 \mathrm{C}$ rate, up to a $40 \%$ improvement in power is predicted for a capacity fraction of $22 \%$ carbon black. For the $2 \mathrm{C}$ rate, up to a $23 \%$ improvement in power is predicted for a capacity fraction of $24 \%$ carbon black. Note that replacement of $60 \%$ carbon black would result in a $6.5 \%$ decrease in the theoretical energy density of the electrode.

The simulated results in Figure 10 were obtained assuming minimal concentration variations within the electrolyte. However, at the high rates used in this study, it is likely that mass transport limitations could exist within this electrode, which may affect the simulated results. Despite this fact, the conclusion that carbon black will improve the pulse power is still valid because the presence of mass transport limitations would only provide another advantage for keeping the reaction front near the separator during the high current pulse. Therefore, the simulated results may slightly underestimate the improvements in pulse-power performance.

This case study was conducted using carbon black as the second electrochemically active material. Over the last twenty years, many different carbonaceous materials have been investigated for use in the lithium-ion negative electrodes (e.g., carbon fibers, petroleum coke, and activated carbon). ${ }^{21,24-26}$ It is likely that the pulse-power performance of the negative electrode can be optimized with the use of another carbon material (or blends of several carbons) as the second electrochemically active material.

\section{Conclusions}

The addition of a second electrochemically active material to a battery electrode is shown to improve the pulse-power performance at high DoD. For both the positive electrode of a sodium metal-halide battery and the negative electrode of a lithium-ion battery, improvements in performance are associated with a decrease in the ionic resistance due to movement of the reaction front toward the separator during pulse-power operation. Under simulated conditions, the maximum power of the sodium metal-halide electrode is predicted to improve by up to $40 \%$ when replacing a fraction of the nickel chloride capacity with iron chloride. Similar improvements in performance for the lithium-ion electrode were predicted when replacing a fraction of the graphite capacity with carbon black.

The impact of the second electrochemically active material on the pulse-power performance was shown to depend highly on the fraction of second active material, the relative importance of ohmic-to-charge transfer resistances $\left(w_{T}\right)$, and the ratio of kinetic parameters between the first and second active materials $(\xi)$. Improvements in the pulse power were predicted for low $w_{T}$, which corresponds to electrodes 
with high ionic resistance relative to charge transfer resistance. Additionally, simulations suggested there is an optimal value of $\xi$ that will maximize the pulse-power of the electrode. Therefore, the electrochemical properties of the second active material should be good enough relative to the first active material (high $\xi$ ) to maximize performance during the pulse discharge while poor enough (low $\xi$ ) to avoid reaction at low DoD.

\section{Acknowledgments}

The sodium metal-halide work was conducted with support from the National Science Foundation Graduate Research Fellowship under grant No. DGE-11-44155. Any opinions, findings, and conclusions or recommendations expressed in this material are those of the authors and do not necessarily reflect the views of the National Science Foundation. The lithium-ion case study was conducted as part of the Center for Mesoscale Transport Properties, an Energy Frontier Research Center supported by the U. S. Department of Energy, Office of Science, Basic Energy Sciences, under award \#DE-SC0012673. The computing was performed on the Yeti Shared HPC Cluster at Columbia University, which includes support from Empire State Development's Division of Science, Technology, and Innovation under contract number C090171.

\section{List of Symbols}

$a$ electrolyte conductivity $\left(\mathrm{S} \mathrm{cm}^{-1}\right)$ density $\left(\mathrm{g} \mathrm{cm}^{-3}\right)$ electrode conductivity $\left(\mathrm{S} \mathrm{cm}^{-1}\right)$ potential in the electrolyte (V)

\section{Subscripts}

denotes first active material denotes second active material denotes solid electrode phase denotes electrolyte phase denotes active material $I$ or $I I$ denotes value during high current pulse denotes reaction

\section{References}

1. U.S. Department of Energy Vehicle Technologies Program: Battery Test Manual for Electric Vehicles, United States Department of Energy, Office of Energy Efficiency and Renewable Energy, Vehicle Technologies Office, INL/EXT-15-34184 (2015).

2. K. G. Gallagher, S.-H. Kang, S. U. Park, and S. Y. Han, 196, 9702 (2011).

3. Battery Requirements for Plug-in Hybrid Electric Vehicles - Analysis and Rationale, National Renewable Energy Laboratory, NREL/CP-540-42240 (2009).

4. M. Doyle, T. F. Fuller, and J. Newman, J. Electrochem. Soc., 140, 1526 (1993).

5. M. Doyle, J. Newman, A. S. Gozdz, C. N. Schmutz, and J. M. Tarascon, J. Elec trochem. Soc., 143, 1890 (1996)

6. J. Rijssenbeek, Y. Gao, Z. Zhong, M. Croft, N. Jisrawi, A. Ignatov, and T. Tsakalakos, J. Power Sources, 196, 2332 (2011).

specific surface area $\left(\mathrm{cm}^{2} \mathrm{~cm}^{-3}\right)$

initial capacity fraction of active material in the electrode

Faraday's constant $\left(96,485 \mathrm{C} \mathrm{mol}^{-1}\right)$

applied current density $\left(\mathrm{A} \mathrm{cm}^{-2}\right)$

baseline current density during discharge $\left(\mathrm{A} \mathrm{cm}^{-2}\right)$

exchange current $\left(\mathrm{A} \mathrm{cm}^{-2}\right)$

current density during high power pulse $\left(\mathrm{A} \mathrm{cm}^{-2}\right)$

reaction rate $\left(\mathrm{A} \mathrm{cm}^{-2}\right)$

electrode thickness $(\mathrm{cm})$

molar mass $\left(\mathrm{g} \mathrm{mol}^{-1}\right)$

number of electron equivalents per mole

volumetric capacity $\left(\mathrm{C} \mathrm{cm}^{-3}\right)$

ideal gas constant $\left(8.314 \mathrm{~J} \mathrm{~mol}^{-1} \mathrm{~K}^{-1}\right)$

time (s)

temperature $(\mathrm{K})$

open circuit potential $(\mathrm{V})$

distance from separator $(\mathrm{cm})$

\section{Greek}

charge transfer coefficient

volume fraction in the electrode

ratio of volumetric discharge capacity of the material to initial volumetric discharge capacity of the material $\left(\left[\mathrm{C} \mathrm{cm}^{-3}\right] /\left[\mathrm{C} \mathrm{cm}^{-3}\right]_{0}\right)$

7. J. L. Sudworth, J. Power Sources, 100, 149 (2001)

8. R. C. Galloway and S. Haslam, J. Power Sources, 80, 164 (1999)

9. P. Albertus, J. Christensen, and J. Newman, J. Electrochem. Soc., 156, A606 (2009).

10. Y. Dai, L. Cai, and R. E. White, J. Power Sources, 247, 365 (2014).

11. S. Basu, R. S. Patil, S. Ramachandran, K. S. Hariharan, S. M. Kolake, T. Song, D. Oh, T. Yeo, and S. Doo, J. Power Sources, 283, 132 (2015).

12. S. Jung, J. Power Sources, 264, 184 (2014).

13. D. Kehrwald, P. R. Shearing, N. P. Brandon, P. K. Sinha, and S. J. Harris, J. Electrochem. Soc., 158, A1393 (2011).

14. J. Newman and K. E. Thomas-Alyea, Electrochemical Systems, Wiley Interscience, Hoboken, New Jersey (2004).

15. A. C. West, Electrochemistry and Electrochemical Engineering: An Introduction (2012).

16. R. Zhu, M. Vallance, S. K. Rahimian, and A. C. West, J. Electrochem. Soc., 162 , A2051 (2015).

17. ASM Ready Reference: Electrical and Magnetic Properties of Metals, ASM International, United States of America (2000).

18. M. Ender, J. Joos, A. Weber, and E. Ivers-Tiffée, J. Power Sources, 269, 912 (2014).

19. M. Ender, J. Power Sources, 282, 572 (2015).

20. R. M. Gnanamuthu and C. W. Lee, Mater. Chem. Phys., 130, 831 (2011).

21. T. F. Fuller, M. Doyle, and J. Newman, J. Electrochem. Soc., 141, 1 (1994)

22. M. Winter, P. Novák, and A. Monnier, J. Electrochem. Soc., 145, 428 (1998).

23. C. Wang, A. J. Appleby, and F. E. Little, J. Electroanal. Chem., 497, 33 (2001).

24. A. Mabuchi, K. Tokumitsu, H. Fujimoto, and T. Kasuh, J. Electrochem. Soc., 142, 1041 (1995).

25. M. W. Verbrugge and B. J. Koch, J. Electrochem. Soc., 143, 600 (1996).

26. L. Zhang, M. Zhang, Y. Wang, Z. Zhang, G. Kan, C. Wang, Z. Zhong, and F. Su, J. Mater: Chem. A, 2, 10161 (2014).

27. K. S. Mohandas, N. Sanil, and P. Rodriguez, Trans. Inst. Min. Metall., Sect. C, 115, 25 (2006). 